\title{
Explicit Solution of the Optimal Reinsurance-Investment Problem with Promotion Budget
}

\author{
Delei SHENG \\ Faculty of Applied Mathematics, Shanxi University of Finance \& Economics, Taiyuan 030006, China \\ E-mail: tjhbsdl@126.com
}

\begin{abstract}
The lack of surrendering profits to policy holder leads to the development of this paper. For an insurer with promotion budget, both the interests of the insurance company and policy-holder are given a balance. In addition, promotion budget is introduced into the risk management process, which makes cheap reinsurance more fair. This article aims at obtaining the explicit strategy and value function for an investment-reinsurance problem under stochastic interest rates. Applying stochastic control technique, a Hamilton-Jacobi-Bellman (HJB) equation is established. The closed-form solution for the HJB equation and a verification theorem are obtained. At last, some numerical analysises illustrate the impacts of different parameters.
\end{abstract}

Keywords promotion budget; stochastic interest rates; power utility; HJB equation

\section{Introduction}

Sales promotion is actually a very common phenomenon in the field of modern insurance. All kinds of promotion activities must have the corresponding promotional budget. In addition, insurance company can't only maximize their own profit, they also need to surrender part of the profits to policy holder, the good form of which would be promotion activity or reducing premiums directly. To sum it up, the insurance company should have the promotional budget.

Usually people require reinsurer's safety loading $\eta>0$ is higher than the insurer's safety loading $\theta>0$ in studying the optimal investment-reinsurance problem. In fact, when insurance company has a promotional budget, the actual premium income of insurer is lower than premiums charged from policy holder (because the actual premium income of insurer is the premium after promotional budget being deducted). In this case, if still require safety loading $\eta>\theta$ is not fair. So it is always taking $\eta=\theta$ in this paper.

Portfolio optimization is a very important research problem, but the study of stochastic portfolio optimization with stochastic interest rates is about just two decades. In 2002, Korn and $\mathrm{Kraft}^{[1]}$ considered investment problems under a stochastic interest rate, where an investor can invest in a savings account, stocks, and bonds and tries to maximize her utility from terminal wealth without the usual Lipschitz assumptions. Assuming the interest rates following the CoxIngersoll-Ross (CIR) dynamics, Grasselli ${ }^{[2]}$ studied an investment problem for the HARA utility functions in 2003. When the interest rate varies according to an ergodic Markov diffusion

Received August 31, 2015, accepted January 25, 2016

Supported by National Natural Science Foundation of China (11301376) 
process, Pang ${ }^{[3]}$ considered a portfolio optimization problem on an infinite time horizon by maximizing the infinite horizon expected discounted log utility of consumption in 2006. Li and $\mathrm{Wu}^{[4]}$ obtained a closed-form expression of the optimal policy that maximizes a power utility for the optimal investment problem with the stochastic interest rate described by a CIR model in 2009. In a market composed of three assets: Cash, stocks and a zero coupon bond, Hainaut ${ }^{[5]}$ investigated the problem of dynamic asset allocation with the dynamics of the instantaneous short rates being driven by a Hull-White model. When interest rates and the inflation rate are stochastic, Munk and Rubtsov ${ }^{[6]}$ solved a stock-bond-cash portfolio problem of a risk-and ambiguity-averse investor in closed form in 2013.

In this paper, we present four main results. We first introduce promotion budget into the risk management process under stochastic interest rates in order to study the interaction of premium and income from investment. We also give the optimal reinsurance strategy and investment strategy, respectively, according to the first-order necessary condition of maximum. In the third part, we obtain the optimal value function by plugging a reasonable conjecture into the HJB equation and solving linear second order partial differential equations. Last but not least, we give a verification theorem and compare the optimal strategies of constant interest rate with the results of stochastic interest rate, a special case of an insurer with promotion budget under a constant interest rate being studied. Finally, we present some numerical examples to illustrate the impacts of parameters in our models.

The remainder of this paper is organized as follows. In Section 2, we formulate a general framework and give some models will be used hereinafter. In Section 3, a Hamilton-JacobiBellman equation is established using stochastic control technique and the explicit solution has been given with a constant relative risk aversion function. Section 4 provides some numerical analysis. Section 5 concludes this paper.

\section{Formulation of the Model}

Throughout this paper, $\left(\Omega, \mathscr{F}, P,\left\{\mathscr{F}_{t}\right\}_{0 \leq t \leq T}\right)$ denotes a complete probability space satisfying the usual condition, where $T>0$ is a finite constant representing the investment time horizon; $\mathscr{F}_{t}$ stands for the information available until time $t$. We also assume the insurer choose cheap reinsurance, i.e., $\eta=\theta$. In the rest of the article you will see that "cheap reinsurance is not really cheap". $\eta=\theta$ is reasonable because the promotion budget deducted directly from insurer's pure premium, which eliminates the arbitrage opportunities even though the safety loadings of insurer and reinsurer are equal.

\subsection{The Financial Market}

We consider a financial market in which transaction amounts are so small that we can consider they have no influence on the prices, and the market with no arbitrage, frictionless, and continuously open as well.

Without loss of generality, we assume that the financial market is composed of two kinds of assets: Cash and equities. For the sake of simplicity, we will only consider one equity asset which can indeed represent the index of a stock market.

The cash asset gives an instantaneous risk-free rate $r(t)$, which diffusion is an Ho and Lee 
$\operatorname{model}^{[7]}$

$$
\mathrm{d} r(t)=a(t) \mathrm{d} t+b \mathrm{~d} \widehat{W}(t), \quad r(0)=r_{0}>0,
$$

where $\widehat{W}(t)$ is a standard Brownian motion, $a(t)>0$ is a real-valued function and the constant $b>0$. Here, we also can use the Vasicek model ${ }^{[8]}$ to get completely similar results.

With an initial value of 1 , the price $S_{0}(t)$ of the cash asset is then given by the following equation:

$$
\frac{\mathrm{d} S^{0}(t)}{S^{0}(t)}=r(t) \mathrm{d} t, \quad S^{0}(0)=1 .
$$

Here $r(t)$ is the stochastic interest rate described by (1).

The stock market index price $S(t)$ at time $t \geq 0$ can be described by the model

$$
\frac{\mathrm{d} S(t)}{S(t)}=[r(t)+u(t)] \mathrm{d} t+v \mathrm{~d} \widetilde{W}(t), \quad S(0)=s_{0},
$$

where $u(t)$ is another positive real-valued function, and the constant $v>0$ denotes the volatility rate of risk asset. Two Brownian motions satisfying $E[\widehat{W}(t), \widetilde{W}(t)]=\rho_{2} \cdot t, \rho_{2} \in[-1,1]$ is the correlation coefficient of $\widehat{W}(t)$ and $\widetilde{W}(t)$.

In the financial model $(3), u(t)>0$ is actual a vital hypothesis, which shows that the development of economy is in good condition. In addition, $u(t)>0$ means that the investment on stock can make more money than investing on cash asset, which is consistent with the economic principle of high-risk high-yield. Only when $u(t)>0$, more people are willing to invest on equities.

\subsection{The Surplus Process of Insurer}

In this paper, we use the classical Cramer-Lunderburg model to describe the surplus process of insurer.

$$
\mathrm{d} R(t)=c \mathrm{~d} t-\mathrm{d}\left(\sum_{i=1}^{N(t)} Y_{i}\right)
$$

where $N(t)$ is a homogeneous Poisson process with intensity $\lambda$, the claim sizes $\left\{Y_{i}, i \geq 1\right\}$ are independent and identically distributed positive random variables with $E\left[Y_{i}\right]=\mu$ and $E\left[Y_{i}^{2}\right]=\sigma^{2}$. According to the expected value premium principle, the pure premium rate of insurer is $c=(1+\theta) \lambda \mu$ and $\theta>0$ is the safety loading of insurer.

The diffusion approximation of the compound poisson process $\mathrm{d}\left(\sum_{i=1}^{N(t)} Y_{i}\right)$ is given by

$$
\mathrm{d}\left(\sum_{i=1}^{N(t)} Y_{i}\right)=\lambda \mu \mathrm{d} t-\sqrt{\lambda} \sigma \mathrm{d} W(t),
$$

where $W(t)$ is a standard Brownian motion and independent with $\widetilde{W}(t)$. But $E[W(t), \widehat{W}(t)]=$ $\rho_{1} \cdot t, \rho_{1} \in[-1,1]$ is the correlation coefficient of $W(t)$ and $\widehat{W}(t)$.

In order to avoid unbearable risk, the insurance company can buy proportional reinsurance to transfer part of the potential risk to reinsurance company. The insurer's retention proportion is $p \in[0,1]$, the remaining $1-p$ shall be borne by the reinsurer. $\eta>0$ is the safety loading of 
reinsurer.

$$
\begin{aligned}
\mathrm{d} R(t) & =[c-(1+\eta)(1-p) \lambda \mu] \mathrm{d} t-p[\lambda \mu \mathrm{d} t-\sqrt{\lambda} \sigma \mathrm{d} W(t)] \\
& =[(1+\theta) \lambda \mu-(1+\eta)(1-p) \lambda \mu-p \lambda \mu] \mathrm{d} t+p \sqrt{\lambda} \sigma \mathrm{d} W(t) .
\end{aligned}
$$

\subsection{The Insurer's Wealth Process with Promotion Budget}

The proportion of wealth invested on stock asset denoted by $\pi(t)$ which is a progressively measurable real function, $X(t)$ with $X(0)=x_{0}$ represents the wealth of the insurer, then we have

$$
\begin{aligned}
\mathrm{d} X(t) & =\mathrm{d} R(t)+\pi(t) X(t) \frac{\mathrm{d} S(t)}{S(t)}+(1-\pi(t)) X(t) \frac{\mathrm{d} S^{0}(t)}{S^{0}(t)} \\
& =[\eta p \lambda \mu+(\pi(t) u(t)+r(t)) X(t)] \mathrm{d} t+p \sqrt{\lambda} \sigma \mathrm{d} W(t)+v \pi(t) X(t) \mathrm{d} \widetilde{W}(t) .
\end{aligned}
$$

Insurer not only seek to maximize their own profit by investment, but also surrender part of the profits to policy holder in the form of budgetary promotion resources, the direct way is to reduce the insurance premiums or hold promotional activities. Thus, the insurer took a fixed share of wealth as a promotional budget, for promotional activities or reduce the insurance premiums directly, to attract more customers. The promotion budget at a fixed experience share deducted from premium income at each time $t$.

$$
\begin{aligned}
\mathrm{d} X(t)= & {[(c-k X(t))-(1+\eta)(1-p) \lambda \mu] \mathrm{d} t-p[\lambda \mu \mathrm{d} t-\sqrt{\lambda} \sigma \mathrm{d} W(t)] } \\
& +\pi(t)(1-k) X(t) \frac{\mathrm{d} S(t)}{S(t)}+(1-\pi(t))(1-k) X(t) \frac{\mathrm{d} S^{0}(t)}{S^{0}(t)} \\
= & \pi(t)(1-k) X(t) \frac{\mathrm{d} S(t)}{S(t)}+(1-\pi(t))(1-k) X(t) \frac{\mathrm{d} S^{0}(t)}{S^{0}(t)} \\
& +[(1+\theta) \lambda \mu-k X(t)-(1+\eta)(1-p) \lambda \mu-p \lambda \mu] \mathrm{d} t+p \sqrt{\lambda} \sigma \mathrm{d} W(t) \\
= & \pi(t)(1-k) X(t)[(r(t)+u(t)) \mathrm{d} t+v \mathrm{~d} \widetilde{W}(t)]+(1-\pi(t))(1-k) X(t) r(t) \mathrm{d} t \\
& +[(1+\theta) \lambda \mu-k X(t)-(1+\eta)(1-p) \lambda \mu-p \lambda \mu] \mathrm{d} t+p \sqrt{\lambda} \sigma \mathrm{d} W(t) \\
= & {[(1-k) X(t) r(t)-k X(t)+\eta p \lambda \mu+\pi(t)(1-k) X(t) r(t) u(t)] \mathrm{d} t } \\
& +\pi(t)(1-k) X(t) v \mathrm{~d} \widetilde{W}(t)+p \sqrt{\lambda} \sigma \mathrm{d} W(t) .
\end{aligned}
$$

We always take $\eta=\theta$ in this paper. The promotional budget deducted directly from the insurer's premium income at time $t$, but the reinsurance premium calculation is based on the premium not deducted the promotional budget. Reinsurance premiums were paid according to the expected premium principle, However, the insurer's premium income is obviously lower than the premium level based on the expected premium principle. Although the safe loading of insurer and reinsurer fixed at the same level, the insurance premium in this paper is also worthy of the phrase: "Cheap reinsurance" is no longer really cheap.

A strategy $\alpha=(p(t), \pi(t))$ is said to be admissible, if $\forall t \in[0, T], \alpha$ is $\mathscr{F}(t)$ progressively measurable and $E\left[\int_{0}^{\infty} \pi^{2}(t) X^{2}(t)\right]<\infty, p(t) \in[0,1], \pi(t) \in[0,+\infty)$. $\Lambda$ denotes the set of all admissible strategies and (8) has a unique (strong) solution. Suppose the insurer has a utility 
function $U(x)$ which is strictly concave and continuously differentiable on $(-\infty,+\infty)$ and aims to maximize the expected utility of his/her terminal wealth.

\section{Main Results}

\subsection{An Insurer with Promotion Budget Under the Stochastic Interest Rate}

In this subsection, we consider the reinsurance-investment problem with stochastic interest rate. Following Merton, we use an expected utility maximization criterion and assume that the insurer's objective is to maximize the expected utility from terminal wealth, where the utility $U(x)$ is a power utility (constant relative risk aversion) function

$$
U(x)=x^{\delta},
$$

the constant $\delta \in(0,1)$ is the relative risk aversion coefficient of a risk averse insurer. This utility function plays a vital role in actuarial mathematics and insurance practice. For an admissible strategy $\alpha=(p(t), \pi(t))$, the value function $J^{\alpha}(t, x, r)$ from state $(x, r)$ at time $t$ is defined as

$$
J^{\alpha}(t, x, r)=E[U(X(T)) \mid X(t)=x, r(t)=r],
$$

and the optimal value function is given by

$$
H(t, x, r)=\max _{(\pi, p)} J^{\alpha}(t, x, r),
$$

with the boundary condition

$$
H(T, x, r)=x^{\delta} .
$$

The goal of the insurer is to find an optimal strategy $\alpha^{*}=\left(p^{*}(t), \pi^{*}(t)\right)$ such that $J^{\alpha^{*}}(t, x, r)=$ $H(t, x, r)$, where $p^{*}(t)$ is called the optimal reinsurance strategy and $\pi^{*}(t)$ is called the optimal investment strategy.

For any $H(t, x, r) \in C^{1,2,2}\left([0, T] \times R_{+} \times R_{+}\right)$, define the generator is a variational operator as the following:

$$
\begin{aligned}
\mathscr{A} H^{\alpha}(t, x, r)= & H_{t}+H_{x}[r(1-k) x-k x]+H_{r} a(t)+H_{r r} \frac{1}{2} b^{2} \\
& +\left\{H_{x} p \eta \lambda \mu+H_{x r} \rho_{1} b p \sqrt{\lambda} \sigma+H_{x x} \frac{1}{2} p^{2} \lambda \sigma^{2}\right\} \\
& +\left\{H_{x} \pi u(t)(1-k) x+H_{x r} \rho_{2} b v \pi(1-k) x+H_{x x} \frac{1}{2} v^{2} \pi^{2}(1-k)^{2} x^{2}\right\},
\end{aligned}
$$

where $H_{t}, H_{x}, H_{r}, H_{x x}, H_{r r}$ and $H_{x r}$ denote the corresponding first and second-order partial derivatives with respect to (w.r.t.) the corresponding variables $t, x, r$, respectively. Similar to Fleming and Soner ${ }^{[9]}$, we can derive the HJB equation for problem (10):

$$
\sup _{\alpha \in \Lambda}\left\{\mathscr{A} H^{\alpha}(t, x, r)\right\}=0
$$

with the boundary condition (11).

In the remainder of this section, we try to find the explicit solutions to problem (10) with stochastic interest by stochastic control technique. 
Theorem 1 For the optimal control problem (10), assume that the objective is to maximize utility of terminal wealth, at the fixed terminal time $T$, and the power utility function is given by

$$
U(x)=x^{\delta}, \quad 0<\delta<1
$$

then the optimal value function is given by

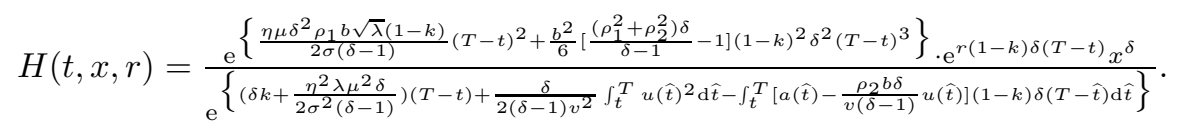

The optimal investment strategy is

$$
\pi^{*}=-\frac{u(t)}{v^{2}(1-k)(\delta-1)}-\frac{\rho_{2} b \delta(T-t)}{v(\delta-1)},
$$

and the optimal reinsurance strategy is

$$
p^{*}=-\frac{\eta \lambda \mu x+\rho_{1} b \sqrt{\lambda} \sigma x(1-k) \delta(T-t)}{\lambda \sigma^{2}(\delta-1)} .
$$

where $\rho_{1} \in[-1,1], \rho_{2} \in[-1,1], b>0, v>0, \mu>0, \lambda>0, \sigma>0$ and $\eta=\theta>0$ are constants, and $u(t)>0, a(t)>0$ are deterministic real functions.

Proof See Appendix.

Remark 1 From the optimal investment strategy (15)

$$
\pi^{*}=-\frac{u(t)}{v^{2}(1-k)(\delta-1)}-\frac{\rho_{2} b \delta(T-t)}{v(\delta-1)}=\frac{1}{v(1-\delta)}\left[\frac{1}{(1-k)} \cdot \frac{u(t)}{v}+b \cdot \rho_{2} \delta(T-t)\right]
$$

where $u(t)>0$ is a positive real-valued function and the constant $b>0$ is the volatility of interest rate. The proportion $\frac{u(t)}{v}$ is excess-appreciate-rate of stock divided by the volatility rate of stock.

Observed carefully from the model (3) and (2), we conclude that $u(t)$ is the excess-appreciaterate of stock relative to cash. The principal parts of the optimal investment strategy $\pi^{*}$ are two components: One is the relative proportion $\frac{u(t)}{v}$, the other is the volatility of interest rate $b$. Because the remainder parts $\frac{1}{(1-k)}$ and $\rho_{2} \delta(T-t)$ are usually fixed in a specific insurer's implementing process. In addition, both of them are so small actually.

In other words, the optimal investment strategy $\pi^{*}$ is mainly determined by two components: The relative ratio of stock's excess-appreciate-rate divided by the stock's volatility rate and the volatility rate of stochastic interest.

Remark 2 From the optimal reinsurance strategy (16), if we let $m=\lambda \mu$ which represents mean-value of insurer's claim size and $n=\sqrt{\lambda} \sigma$ which denotes variance of insurer's claim size, then

$$
\begin{aligned}
p^{*} & =-\frac{\eta \lambda \mu x+\rho_{1} b \sqrt{\lambda} \sigma x(1-k) \delta(T-t)}{\lambda \sigma^{2}(\delta-1)} \\
& =\frac{\eta m x+\rho_{1} b n x(1-k) \delta(T-t)}{n^{2}(1-\delta)} \\
& =x \cdot\left[\frac{\eta}{(1-\delta)} \cdot \frac{m}{n^{2}}+\frac{\rho_{1} b}{n} \cdot(1-k) \frac{\delta}{(1-\delta)}(T-t)\right] .
\end{aligned}
$$


It's nature that the optimal reinsurance strategy $p^{*}$ increases with respect to the wealth level at time $t$. The most interesting discovery is that we find the optimal reinsurance strategy mainly determined by $\frac{m}{n^{2}}$ and $\frac{\rho_{1} b}{n}$. Because $\frac{\eta}{(1-\delta)}$ and $(1-k) \frac{\delta}{(1-\delta)}(T-t)$ are usually fixed in a specific insurer's implementing process. In fact, $\frac{\rho_{1} b}{n}$ shows us the correlation of insurer's claim volatility and the volatility of interest rate. $\frac{m}{n^{2}}$ is the proportion for mean and variance of insurer's claim size. After analyzing carefully, we conclude that the volatility of insurer's claim size is the origin force to change of the optimal reinsurance strategy $p^{*}$.

Theorem 2 (verification theorem) Let $H(t, x, r)$ be a convex, twice differential solution to (13) with the boundary condition (11) such that $\left|H\left(0, x_{0}, r_{0}\right)\right|<\infty$. Then, for all $t \in$ $[0, T], x \in(0, \infty)$ :

(I) $H(t, x, r) \leq J^{\alpha}(t, x, r)$ for every admissible strategy $\alpha$.

(II) If there exists an admissible control $\alpha^{*}=\left(p^{*}, \pi^{*}\right)$ such that

$$
\alpha^{*} \in \arg \max \left[\mathscr{A}^{\alpha} H\left(t, x^{*}(t), r^{*}(t)\right)\right] .
$$

Then the value function $H(t, x, r)=J^{\alpha^{*}}(t, x, r)$ and the policy $\alpha^{*}=\left(p^{*}, \pi^{*}\right)$ is the optimal strategy corresponding to $X^{\alpha^{*}}(t)$ which is the solution of (8).

Proof The proof is classical, refer to Fleming and Soner ${ }^{[9]}$, Guan and Liang ${ }^{[10,11]}$ and He and Liang ${ }^{[12]}$, so we omit it here.

\subsection{A Special Case of an Insurer with Promotion Budget Under a Constant In- terest Rate}

In this subsection, we investigate a special case that the interest rate $r(t)$ is a constant $r$. All assumptions are the same as above except that $r(t) \equiv r$ throughout this part, thus we have

$$
\frac{\mathrm{d} S^{0}(t)}{S^{0}(t)}=r \mathrm{~d} t, \quad S^{0}(0)=1,
$$

and

$$
\frac{\mathrm{d} S(t)}{S(t)}=[r+u(t)] \mathrm{d} t+v \mathrm{~d} \widetilde{W}(t), \quad S(0)=s_{0},
$$

where $r, v$ are positive constants, $u(t)$ is a positive real-valued function. The surplus processes with claim and reinsurance are the same as (5) and (6), $W(t)$ and $\widetilde{W}(t)$ are independent standard Brownian motions. So we get the wealth process with promotion budget and investing in the financial market as follows:

$$
\begin{aligned}
\mathrm{d} X(t)= & \mathrm{d} R(t)-k X(t) \mathrm{d} t+\pi(t)(1-k) X(t) \frac{\mathrm{d} S(t)}{S(t)}+(1-\pi(t))(1-k) X(t) \frac{\mathrm{d} S^{0}(t)}{S^{0}(t)} \\
= & {[(1-k) X(t) r-k X(t)+\eta p \lambda \mu+\pi(t)(1-k) X(t) r u(t)] \mathrm{d} t } \\
& +\pi(t)(1-k) X(t) v \mathrm{~d} \widetilde{W}(t)+p \sqrt{\lambda} \sigma \mathrm{d} W(t) .
\end{aligned}
$$

we consider the utility $U(x)$ is a power utility (constant relative risk aversion) function

$$
U(x)=x^{\delta}
$$

to maximize the expected utility of terminal wealth, where the constant $\delta \in(0,1)$ is the relative risk aversion coefficient of a risk averse insurer. For an admissible strategy $\alpha=(p(t), \pi(t))$, the value function $J^{\alpha}(t, x, r)$ from state $x$ at time $t$ is defined as 


$$
J^{\alpha}(t, x)=E[U(X(T)) \mid X(t)=x],
$$

and the optimal value function is given by

$$
H(t, x)=\max _{(\pi, p)} J^{\alpha}(t, x)
$$

with the boundary condition $H(T, x, r)=x^{\delta}$.

Applying the classical tools of stochastic optimal control, if the optimal value function $H(t, x) \in C^{1,2}([0, T] \times R)$, then $H$ satisfies the following HJB equation:

$$
\begin{aligned}
& H_{t}+H_{x}[r-(1+r) k] x+\max _{p}\left\{H_{x} p \eta \lambda \mu+H_{x x} \frac{1}{2} p^{2} \lambda \sigma^{2}\right\} \\
& +\max _{\pi}\left\{H_{x} \pi u(t)(1-k) x+H_{x x} \frac{1}{2} v^{2} \pi^{2}(1-k)^{2} x^{2}\right\}=0 .
\end{aligned}
$$

According to the first order necessary condition of extreme, we obtain that

$$
p^{*}=-\frac{\eta \mu H_{x}}{\sigma^{2} H_{x x}}
$$

and

$$
\pi^{*}=-\frac{u(t) H_{x}}{v^{2}(1-k) x H_{x x}} .
$$

Conjecture of the solution has the following form

$$
\left\{\begin{array}{l}
H(t, x)=f(t) x^{\delta} \\
H(T, x)=x^{\delta}, \quad 0<\delta<1, \\
f(T)=1 .
\end{array}\right.
$$

The derivatives of the conjecture with respect to $t, x$, respectively.

$$
H_{t}=f^{\prime}(t) x^{\delta}, \quad H_{x}=f(t) \delta x^{\delta-1}, \quad H_{x x}=f(t) \delta(\delta-1) x^{\delta-2} .
$$

Plugging these derivatives into the HJB equation, we have

$$
f^{\prime}(t)+f(t) \delta[r(1-k)-k]-\frac{[\eta \lambda \mu \delta]^{2} f(t)}{2 \lambda \sigma^{2} \delta(\delta-1)}-\frac{[\delta u(t)]^{2} f(t)}{2 v^{2} \delta(\delta-1)}=0 .
$$

The solution of the above ordinary differential equation is

$$
f(t)=\exp \left\{\delta \int_{t}^{T}\left[r+(1+r) k-\frac{\eta^{2} \lambda \mu^{2}}{2 \sigma^{2}(\delta-1)}-\frac{u(\tau)^{2}}{2 v^{2}(\delta-1)}\right] \mathrm{d} \tau\right\} .
$$

We obtain the value function

$$
H(t, x)=x^{\delta} \exp \left\{\left[r+(1+r) k-\frac{\eta^{2} \lambda \mu^{2}}{2 \sigma^{2}(\delta-1)}\right] \delta(T-t)-\delta \int_{t}^{T}\left[\frac{u(\tau)^{2}}{2 v^{2}(\delta-1)}\right] \mathrm{d} \tau\right\},
$$

and the optimal strategy $\alpha^{*}=\left(p^{*}, \pi^{*}\right)$ as follows:

and

$$
p^{*}=\frac{1}{(1-\delta)} \cdot \frac{\eta \mu x}{\sigma^{2}}
$$

$$
\pi^{*}=\frac{u(t)}{v^{2}(1-k)(1-\delta)}
$$


Remark 3 The optimal strategy $\alpha^{*}=\left(p^{*}, \pi^{*}\right)$ with a constant interest rate

$$
p^{*}=\frac{\eta \mu x}{(1-\delta) \sigma^{2}}, \quad \pi^{*}=\frac{u(t)}{v^{2}(1-k)(1-\delta)},
$$

and the optimal strategy $\alpha^{*}=\left(p^{*}, \pi^{*}\right)$ with stochastic interest rate

$$
\begin{aligned}
& p^{*}=\frac{\eta \mu x}{\sigma^{2}(1-\delta)}+\frac{\rho_{1} b}{\sqrt{\lambda} \sigma} \cdot \frac{\delta(T-t)}{(1-\delta)} \cdot(1-k) x, \\
& \pi^{*}=\frac{u(t)}{v^{2}(1-k)(1-\delta)}+\frac{\rho_{2} b}{v} \cdot \frac{\delta(T-t)}{(1-\delta)} .
\end{aligned}
$$

Comparing the results of constant interest rate with the results of stochastic interest rate, it is easy to find that the optimal strategy under the stochastic interest rate exists one term more than the optimal strategy under a constant interest rate.

$$
p^{*} \text { stochastic }-p^{*} \text { constant }=\rho_{1} \cdot \frac{b}{\sqrt{\lambda} \sigma} \cdot \frac{\delta(T-t)}{(1-\delta)} \cdot(1-k) x .
$$

If $\rho_{1}>0$, then $p^{*}$ stochastic $>p^{*}$ constant, else if $\rho_{1}<0$, then $p^{*}$ stochastic $<p^{*}$ constant.

Let's pay attention to $\frac{b}{\sqrt{\lambda} \sigma}$ which is a ratio of interest rate volatility rate divided by the volatility rate of claim size. So in other words, it's a share of risk-free asset volatility relative to the claim size volatility at time $t$.

Similarly,

$$
\pi^{*} \text { stochastic }-\pi^{*} \text { constant }=\rho_{2} \cdot \frac{b}{v} \cdot \frac{\delta(T-t)}{(1-\delta)} .
$$

If $\rho_{2}>0$, then $\pi^{*}$ stochastic $>\pi^{*}$ constant, else if $\rho_{2}<0$, then $\pi^{*}$ stochastic $<\pi^{*}$ constant.

It is noteworthy that $\frac{b}{v}$ is a ratio of interest rate volatility rate divided by the volatility rate of risky assets, in other words, it's a share of risk-free asset volatility relative to the risk assets volatility.

In one word, whether the expression $\rho_{1} \cdot \frac{b}{\sqrt{\lambda} \sigma} \cdot \frac{\delta(T-t)}{(1-\delta)} \cdot(1-k) x$ or the formula $\rho_{2} \cdot \frac{b}{v} \cdot \frac{\delta(T-t)}{(1-\delta)}$ reflects the interest rate volatility's influence on the optimal strategy. However, this influence is a synergy effect based on the three major factors: interest rate volatility, risky asset volatility and claim size volatility. Of course, there may be other factors, such as investor risk aversion, and so on.

\section{Numerical Analysis}

In this section, we give some qualitative results to show the relationship between the optimal control strategies (optimal investment strategy and optimal proportional reinsurance strategy, respectively) and the parameters in our model. Unless otherwise stated, the hypothetical values of model parameters are as follows:

$$
\begin{aligned}
& x=6, \quad \eta=0.1, \quad \lambda=2, \quad \mu=0.2, \quad b=0.05, \quad v=0.1, \\
& k=0.1, \quad T=10, \quad \delta=0.5, \quad \sigma=2, \quad \rho_{1}=0.3, \quad \rho_{2}=0.1 .
\end{aligned}
$$

For simplicity, we also can take the positive real-valued function $u(t)$ and $a(t)$ as positive constants or take other appropriate functions. 
First, let's focus on the optimal reinsurance proportion $p^{*}$ as in (16). Setting $\lambda \mu=m$ and $\sqrt{\lambda} \sigma=n$, then

$$
\begin{aligned}
p^{*} & =-\frac{\eta \lambda \mu x+\rho_{1} b \sqrt{\lambda} \sigma x(1-k) \delta(T-t)}{\lambda \sigma^{2}(\delta-1)} \\
& =-\frac{\eta m x+\rho_{1} b n x(1-k) \delta(T-t)}{n^{2}(\delta-1)} \\
& =\frac{1}{(1-\delta)} \cdot \frac{1}{n} \cdot\left[\frac{\eta m x}{n}+\rho_{1} b \delta(1-k) x(T-t)\right] .
\end{aligned}
$$

Here $m$ says the average claim amount and $-n$ is the volatility of claim amount.

Proposition 1 The relative risk aversion coefficient $0<\delta<1$ for an risk averse insurer and the optimal reinsurance proportion $p^{*}$ given by (17), If $n=\sqrt{\lambda} \sigma$ and $m=\lambda \mu$, then the optimal strategy $p^{*}$ increases with respect to the volatility $-n$ of claim size, the mean value $m$ of claim size and the correlation coefficient $\rho_{1}$, where $\rho_{1}$ representing the correlation of interest rate volatility and claim volatility.

Proof

$$
\frac{\partial p^{*}}{\partial m}=\frac{x \eta}{n^{2}(1-\delta)}>0, \quad \frac{\partial p^{*}}{\partial n}=-\frac{x\left(2 m \eta+b(1-k) n(T-t) \sigma \rho_{1}\right)}{n^{3}(1-\delta)}<0,
$$

which means $p^{*}$ decreases with respect to $n$, so the optimal reinsurance proportion $p^{*}$ increases with respect to $-n$.

$$
\frac{\partial p^{*}}{\partial \rho_{1}}=\frac{b(1-k)(T-t) x \sigma}{n(1-\delta)}>0 .
$$

Remark 4 The equation (5) shows that $m=\lambda \mu$ is the mean value of claim size and $-n=-\sqrt{\lambda} \sigma$ is the volatility of claim size at time $t$. The quantity of both $m$ and $-n$ means risk of an insurer, either of them increasing may lead more risk for an insurer, so the insurer must transfer more risk to the reinsurer. It is very natural that the reinsurance proportion increases with respect to the average claim amount and the volatility of claim size. See Figure 1.

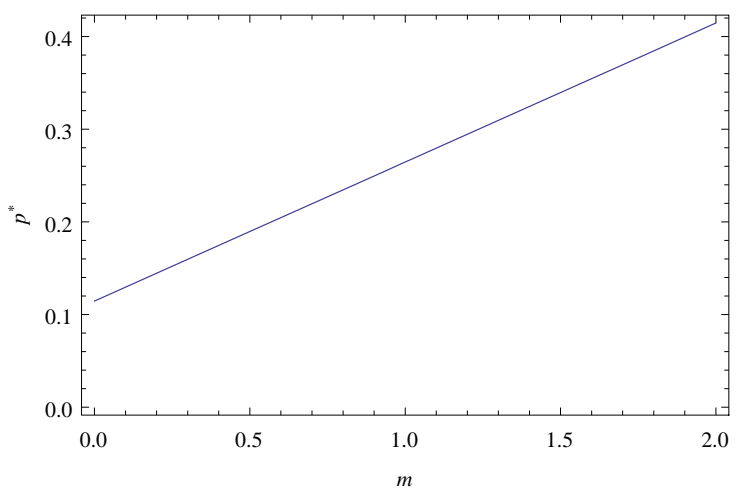

Figure 1 The optimal reinsurance proportion $p^{*}$ varies with $m$

Which would be really interesting is that the optimal reinsurance proportion $p^{*}$ increases with respect to the correlation coefficient $\rho_{1}$ which represents the correlation of interest rate volatility and claim volatility. $\rho_{1}$ is so important a parameter that including analysis of the 
three other parameters $k, b, x$ are first depends on $\rho_{1}$. Simple calculation we can see it as follow: If $\rho_{1}>0$, then

$$
\begin{aligned}
& \frac{\partial p^{*}}{\partial k}=\frac{b(T-t) x \sigma \rho_{1}}{n(1-\delta)}>0, \quad \frac{\partial p^{*}}{\partial b}=\frac{(1-k)(T-t) x \sigma \rho_{1}}{n(1-\delta)}>0, \\
& \frac{\partial p^{*}}{\partial x}=\frac{m \eta+b(1-k) n(T-t) \sigma \rho_{1}}{n^{2}(1-\delta)}>0,
\end{aligned}
$$

else if $\rho_{1}<0$, then

$$
\frac{\partial p^{*}}{\partial k}=\frac{b(T-t) x \sigma \rho_{1}}{n(1-\delta)}<0, \quad \frac{\partial p^{*}}{\partial b}=\frac{(1-k)(T-t) x \sigma \rho_{1}}{n(1-\delta)}<0
$$

but for the case of $\rho_{1}<0, \frac{\partial p^{*}}{\partial x}=\frac{m \eta+b(1-k) n(T-t) \sigma \rho_{1}}{n^{2}(1-\delta)}$ is undetermined.

The mutual influence between $k, b, x$ and $\rho_{1}$, the reason has to speak of from the comparison of the optimal reinsurance proportion $p^{*}$ under the stochastic interest rate with a special case under a constant interest rate in the previous section. The generation of a term contains $\rho_{1}, k, b, x$ in the optimal reinsurance proportion $p^{*}$, which is all because stochastic interest rate is introduced. The intuitive graphical interpretation, please refer to Figure 2, Figure 3, Figure 4 and Figure 5.

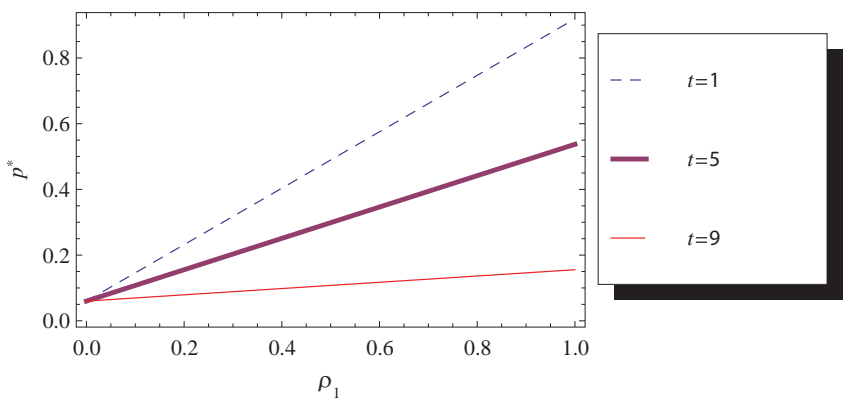

Figure 2 The optimal reinsurance proportion $p^{*}$ with $\rho_{1}$

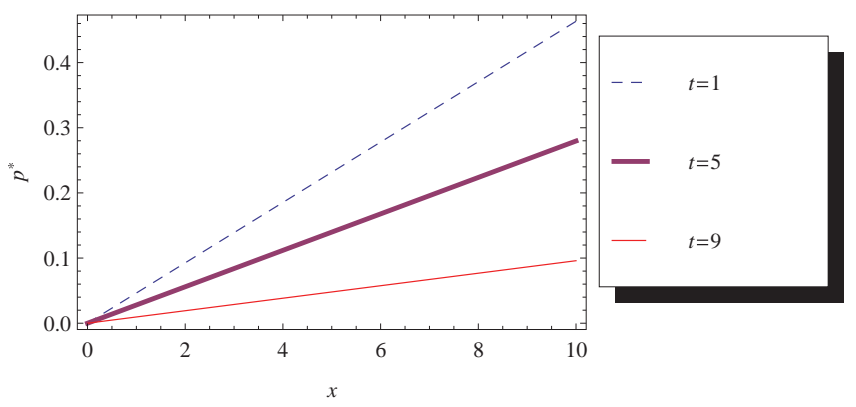

Figure 3 The optimal reinsurance proportion $p^{*}$ with $x$ for $\rho_{1}>0$ 


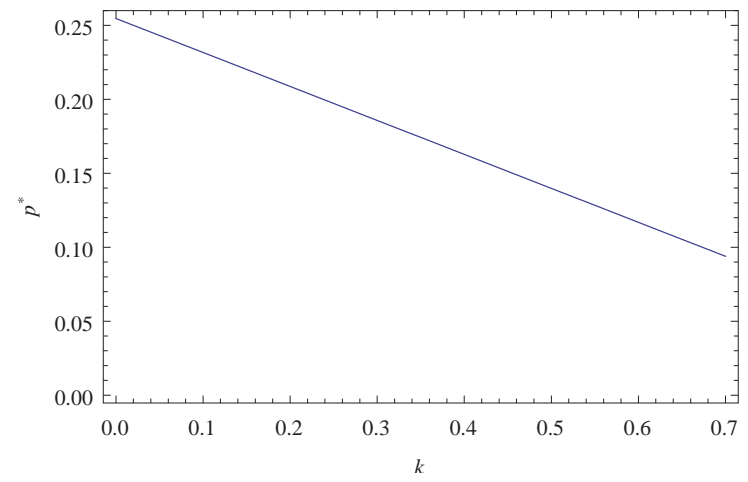

Figure 4 The optimal reinsurance proportion $p^{*}$ varies with $k$

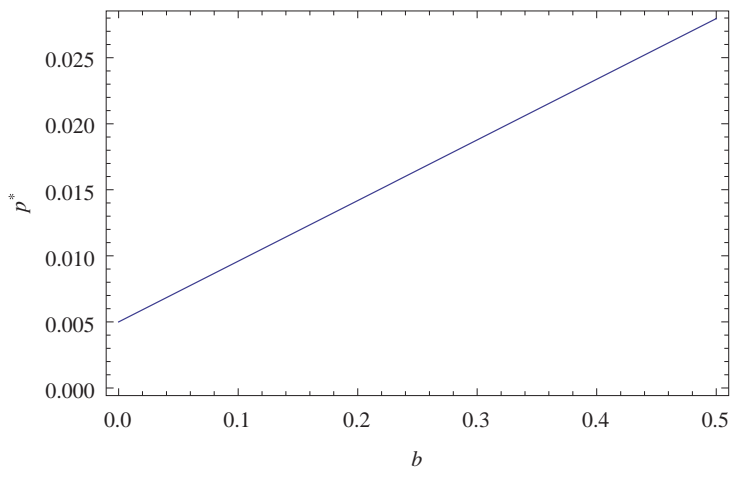

Figure 5 The optimal reinsurance proportion $p^{*}$ varies with $b$

Second, let's pay attention to the optimal investment strategy (15). If $u(t)$ denoted by $z$, then (15) can be rewritten as

$$
\pi^{*}=-\frac{u(t)}{v^{2}(1-k)(\delta-1)}-\frac{\rho_{2} b \delta(T-t)}{v(\delta-1)}=\frac{1}{(1-\delta)} \cdot \frac{1}{v} \cdot\left[\frac{z}{v(1-k)}+\rho_{2} b \delta(T-t)\right] .
$$

Proposition 2 The relative risk aversion coefficient $0<\delta<1$ for an risk averse insurer and the optimal investment strategy $\pi^{*}$ given by (18), then the optimal strategy $\pi^{*}$ increases with respect to the correlation coefficient $\rho_{2}$, the budgetary proportion $k$ and the positive real-valued function $u(t)$, respectively.

Proof

$$
\frac{\partial \pi^{*}}{\partial \rho_{2}}=\frac{b}{v} \cdot \frac{\delta}{(1-\delta)}(T-t)>0, \quad \frac{\partial \pi^{*}}{\partial k}=\frac{u(t)}{(1-k)^{2} v^{2}(1-\delta)}>0,
$$

In order to avoid the influence of $t$, we take the positive real-valued function $u(t)$ as a positive constant $z$ when analyzing changes with the value of $u(t)$.

$$
\frac{\partial \pi^{*}}{\partial z}=\frac{1}{(1-k) v^{2}(1-\delta)}>0 .
$$

Remark 5 Figure 6, Figure 7 and Figure 8 coincide with the above Proposition 2. The promotional budget can be appropriately increased only when the investment income increased, 
so $\frac{\partial \pi^{*}}{\partial k}>0$, the relationship that proportion $\pi^{*}$ of investment increases with budget share $k$ is easy to understand.

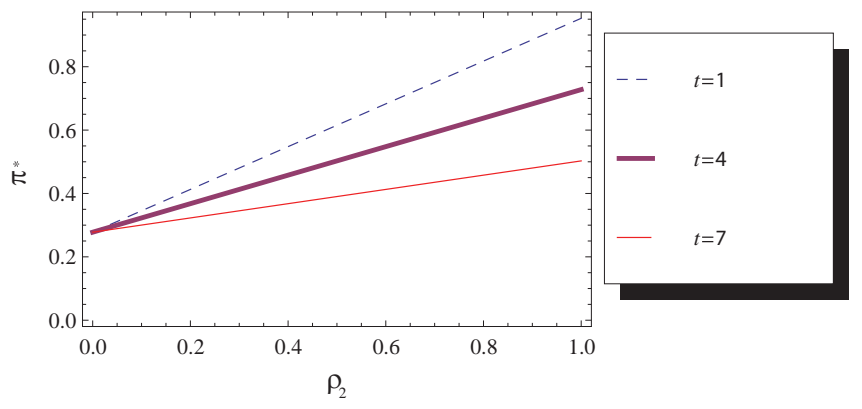

Figure 6 The optimal investment strategy $\pi^{*}$ varies with $\rho_{2}$

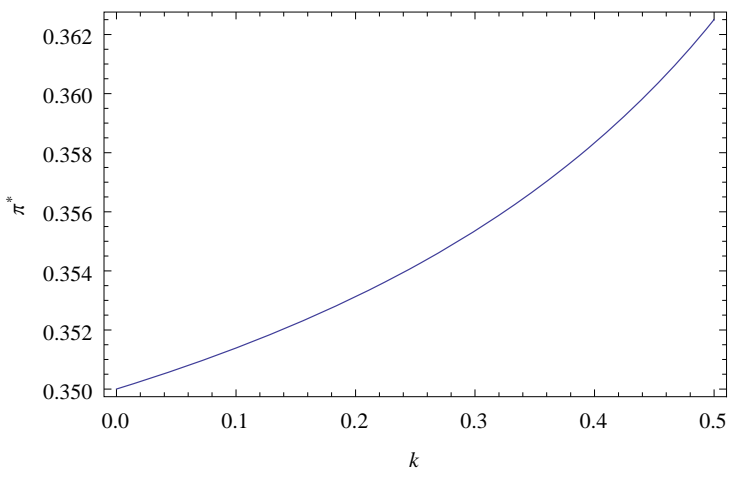

Figure 7 The optimal investment strategy $\pi^{*}$ varies with $k$

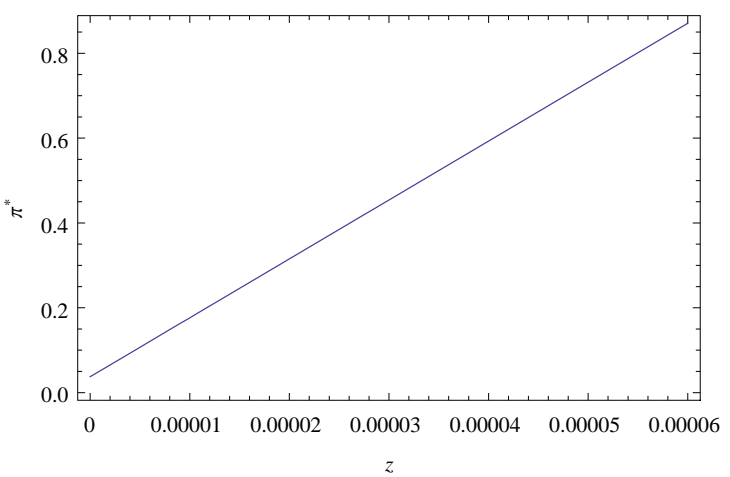

Figure 8 The optimal investment strategy $\pi^{*}$ varies with $z$

Since $z=u(t)$ represents the appreciation rate of risky assets higher than average rate of return from risk-free assets, we call $u(t)$ beyond earning. $\frac{\partial \pi^{*}}{\partial z}>0\left(\frac{\partial \pi^{*}}{\partial u(t)}>0\right)$ shows that the investment proportion of risky asset increases with beyond earning. The more bigger $u(t)$, the more higher income from risk investment than risk-free return, which will attract more money invested on the risk asset and force the investment proportion $\pi^{*}$ increased. 
In the above Proposition 2, one parameter which is worthy paying great attention on is $\rho_{2}$. It is a key parameter, including analysis of the two other parameters $b, v$ are first depends on $\rho_{2}$.

If $\rho_{2}>0$, then

$$
\frac{\partial \pi^{*}}{\partial b}=\frac{(T-t) \delta \rho_{2}}{v(1-\delta)}>0, \text { and } \frac{\partial \pi^{*}}{\partial v}=-\frac{2 u(t)+b(1-k)(T-t) v \delta \rho_{2}}{(1-k) v^{3}(1-\delta)}<0,
$$

else if $\rho_{2}<0$, then

$$
\frac{\partial \pi^{*}}{\partial b}=\frac{(T-t) \delta \rho_{2}}{v(1-\delta)}<0
$$

But for the case of $\rho_{2}<0, \frac{\partial \pi^{*}}{\partial v}=-\frac{2 u(t)+b(1-k)(T-t) v \delta \rho_{2}}{(1-k) v^{3}(1-\delta)}$ is undetermined.

Investigated the reason why $\rho_{2}$ is so important, it has to speak of from the comparison of the optimal investment strategy under the stochastic interest rate and the optimal strategy under a constant interest rate. The generation of a term contains $\rho_{2}, b, v$ in the optimal investment strategy, which is all because stochastic interest rate is introduced. Intuitive graphical illustration refer to Figure 9 and Figure 10.

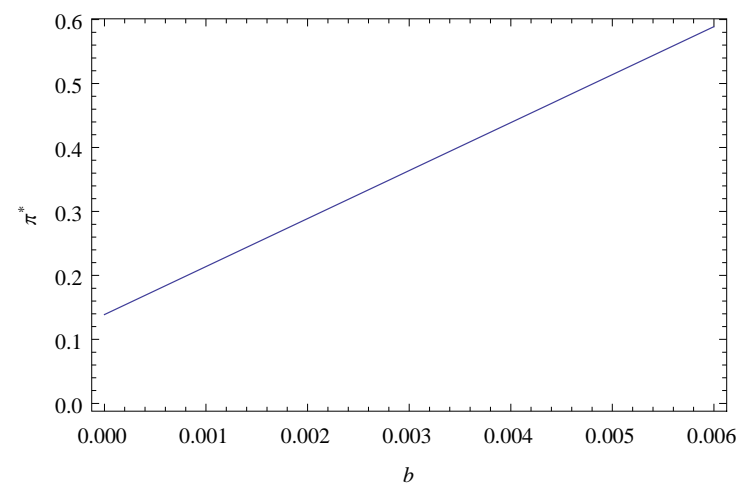

Figure 9 The optimal investment strategy $\pi^{*}$ varies with $b$ for the case of $\rho_{2}>0$

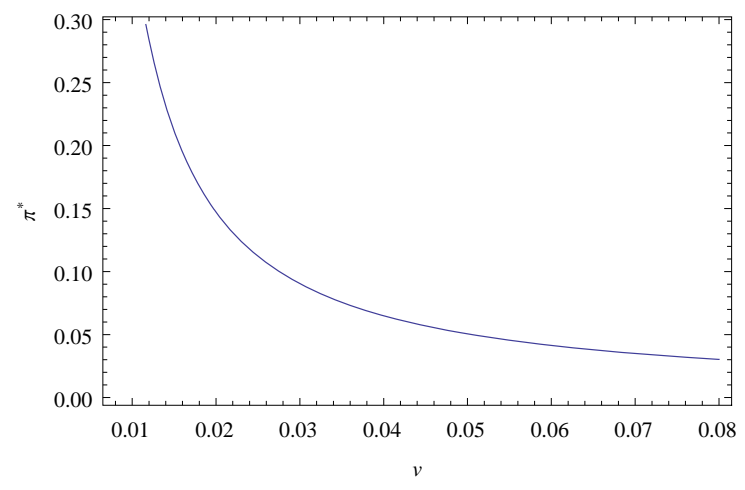

Figure 10 The optimal investment strategy $\pi^{*}$ varies with $v$ for the case of $\rho_{2}>0$ 
Finally, we will show you the numerical analysis of the value function $V(t, x, r)$. For simplicity, we take $a(t)=r+b$ and $u(t)=a(t)+b$ here, because $r(t)$ must take a specific value $r$ at time $t$.

No matter what level of risk aversion or observed at any given time, the utility has been monotone increasing with the increase of wealth levels, but the speed of utility increasing with wealth level is slower and slower. This characteristic is clear at a glance from Figure 11 and Figure 12.

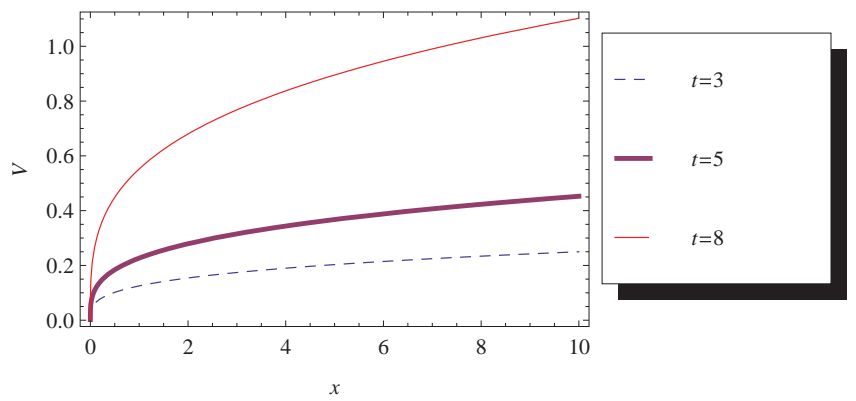

Figure $11 V(t, x, r)$ varies with $x$ for different $t$ at $\delta=0.3$

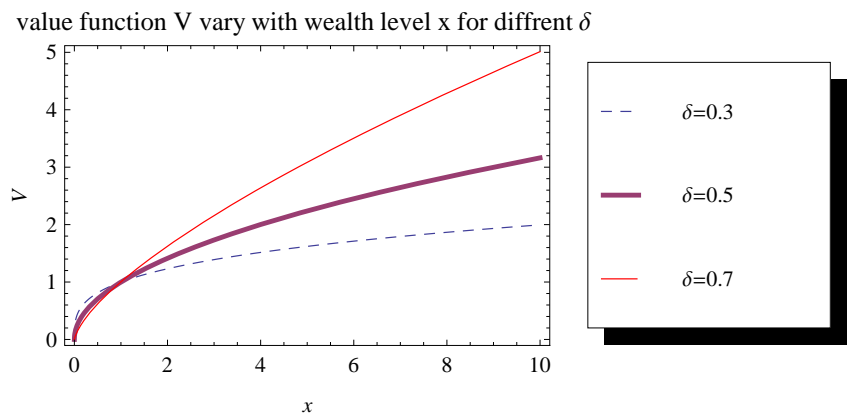

Figure $12 V(t, x, r)$ varies with $x$ for different $\delta$ at $t=10$

\section{Conclusion}

Promotion budget being introduced into the risk management process is more accords with the practice of modern insurance company's operation, which also provides a way to eliminate arbitrage opportunities when the insurance company to buy cheap reinsurance (the case of $\eta=\theta$ ). Almost all literatures on stochastic control problems in insurance consider the control systems without promotion budget. Authors only sought to maximize the insurer's profit, but ignore an important issue of surrendering part of the profits to policy holder. In the practice of the modern economy, promotion is an important means to attract more customers.

\section{References}

[1] Korn R, Kraft H. A stochastic control approach to portfolio problems with stochastic interest rates. SIAM Journal on Control and Optimization, 2002, 40(4): 1250-1269. 
[2] Grasselli M. A stability result for the HARA class with stochastic interest rates. Insurance: Mathematics and Economics, 2000, 33(3): 611-627.

[3] Pang T. Stochastic portfolio optimization with log utility. International Journal of Theoretical and Applied Finance, 2011, 9(6): 869-887.

[4] Li J, Wu R. Optimal investment problem with stochastic interest rate and stochastic volatility: Maximizing a power utility. Applied Stochastic Models in Business and Industry, 2009, 25(3): 407-420.

[5] Hainaut D. Dynamic asset allocation under VaR constraint with stochastic interest rates. Annals of Operations Research, 2009, 172(1): 97-117.

[6] Munk C, Rubtsov A. Portfolio management with stochastic interest rates and inflation ambiguity. Annals of Finance, 2014, 10(3): 419-455.

[7] Ho T S Y, Lee S B. Term structure movements and pricing interest rate contingent claims. Journal of Finance, 1986, 41(5): 1011-1029.

[8] Vasicek O. An equilibrium characterisation of the term structure. Journal of Financial Economics, 1977, 5(2): $177-188$.

[9] Fleming H U, Soner H M. Controlled Markov processes and viscosity solutions. Springer, Berlin, 2010.

[10] Guan G, Liang Z. Optimal reinsurance and investment strategies for insurer under interest rate and inflation risks. Insurance: Mathematics and Economics, 2014, 55(1): 105-115.

[11] Guan G, Liang Z. Optimal management of DC pension plan in a stochastic interest rate and stochastic volatility framework. Insurance: Mathematics and Economics, 2014, 57(6): 58-66.

[12] He L, Liang Z. Optimal financing and dividend control of the insurance company with proportional reinsurance policy. Insurance: Mathematics and Economics, 2008, 42(3): 976-983.

\section{Appendix}

\section{A.1 Proof of Theorem 1}

Proof The HJB equation (13) rewritten by

$$
\begin{aligned}
& H_{t}+H_{x}[r(1-k) x-k x]+H_{r} a(t)+H_{r r} \frac{1}{2} b^{2} \\
& +\max _{p}\left\{H_{x} p \eta \lambda \mu+H_{x r} \rho_{1} b p \sqrt{\lambda} \sigma+H_{x x} \frac{1}{2} p^{2} \lambda \sigma^{2}\right\} \\
& +\max _{\pi}\left\{H_{x} \pi u(t)(1-k) x+H_{x r} \rho_{2} b v \pi(1-k) x+H_{x x} \frac{1}{2} v^{2} \pi^{2}(1-k)^{2} x^{2}\right\}=0 .
\end{aligned}
$$

According to the first-order necessary conditions of the extremum, the optimal strategies

$$
\begin{aligned}
p^{*} & =-\frac{\eta \lambda \mu H_{x}+\rho_{1} b \sqrt{\lambda} \sigma H_{x r}}{\lambda \sigma^{2} H_{x x}}, \\
\pi^{*} & =-\frac{u(t) H_{x}+\rho_{2} b v H_{x r}}{v^{2}(1-k) x H_{x x}} .
\end{aligned}
$$

Inserting (A2) and (A3) into (A1), the HJB equation becomes

$$
\begin{aligned}
& H_{t}+H_{r} a(t)+H_{x}[r(1-k) x-k x]+H_{r r} \frac{1}{2} b^{2} \\
& -\frac{\left[\eta \lambda \mu H_{x}+\rho_{1} b \sqrt{\lambda} \sigma H_{x r}\right]^{2}}{2 \lambda \sigma^{2} H_{x x}}-\frac{\left[u(t) H_{x}+\rho_{2} b v H_{x r}\right]^{2}}{2 v^{2} H_{x x}}=0 .
\end{aligned}
$$


Conjecture of the solution has the following form

$$
\left\{\begin{array}{l}
H(t, x, r)=f(t) \mathrm{e}^{h(t) r} x^{\delta}, \\
H(T, x, r)=x^{\delta}, \quad 0<\delta<1, \\
f(T)=1 \\
h(T)=0 .
\end{array}\right.
$$

The derivatives of the conjecture with respect to $t, x, r$, respectively.

$$
\begin{array}{ll}
H_{t}=\left(f^{\prime}(t)+f(t) h^{\prime}(t) r\right) \mathrm{e}^{h(t) r} x^{\delta}, & H_{x}=f(t) \mathrm{e}^{h(t) r} \delta x^{\delta-1}, \\
H_{r}=f(t) \mathrm{e}^{h(t) r} x^{\delta} h(t), & H_{x x}=f(t) \mathrm{e}^{h(t) r} \delta(\delta-1) x^{\delta-2}, \\
H_{r r}=f(t) \mathrm{e}^{h(t) r} x^{\delta} h(t)^{2}, & H_{x r}=f(t) \mathrm{e}^{h(t) r} \delta x^{\delta-1} h(t) .
\end{array}
$$

Plugging these derivatives into (A4)

$$
\begin{aligned}
& f^{\prime}(t)+f(t) h^{\prime}(t) r+f(t) \delta[r(1-k)-k]+f(t) h(t) a(t) \\
& +\frac{b^{2}}{2} f(t) h(t)^{2}-\frac{\left[\eta \lambda \mu \delta+\rho_{1} b \sqrt{\lambda} \sigma \delta h(t)\right]^{2} f(t)}{2 \lambda \sigma^{2} \delta(\delta-1)}-\frac{\left[\delta u(t)+\rho_{2} b v \delta h(t)\right]^{2} f(t)}{2 v^{2} \delta(\delta-1)}=0 .
\end{aligned}
$$

Splitting into two equations

$$
\left[h^{\prime}(t)+\delta(1-k)\right] f(t) r=0,
$$

i.e.,

$$
\begin{aligned}
& \left\{\begin{array}{l}
h^{\prime}(t)+\delta(1-k)=0 \\
h(T)=0
\end{array}\right. \\
& \left\{\begin{array}{l}
f^{\prime}(t)-f(t) \delta k+f(t) h(t) a(t)+\frac{b^{2}}{2} f(t) h(t)^{2} \\
-\frac{\left[\eta \lambda \mu \delta+\rho_{1} b \sqrt{\lambda} \sigma \delta h(t)\right]^{2} f(t)}{2 \lambda \sigma^{2} \delta(\delta-1)}-\frac{\left[\delta u(t)+\rho_{2} b v \delta h(t)\right]^{2} f(t)}{2 v^{2} \delta(\delta-1)}=0 \\
f(T)=1
\end{array}\right.
\end{aligned}
$$

The solution of (A8)

$$
h(t)=(1-k) \delta(T-t)
$$

Inserting (A10) into (A9),

$$
\begin{aligned}
\frac{f^{\prime}(t)}{f(t)}= & \delta k-a(t)(1-k) \delta(T-t)-\frac{b^{2}}{2}[(1-k) \delta(T-t)]^{2} \\
& +\frac{\left[\eta \lambda \mu \delta+\rho_{1} b \sqrt{\lambda} \sigma \delta(1-k) \delta(T-t)\right]^{2}}{2 \lambda \sigma^{2} \delta(\delta-1)}+\frac{\left[\delta u(t)+\rho_{2} b v \delta(1-k) \delta(T-t)\right]^{2}}{2 v^{2} \delta(\delta-1)}
\end{aligned}
$$


$f(T)=1$.

The solution of (A11)

$$
f(t)=\frac{\mathrm{e}^{\left\{\frac{\eta \mu \delta^{2} \rho_{1} b \sqrt{\lambda}(1-k)}{2 \sigma(\delta-1)}(T-t)^{2}+\frac{b^{2}}{6}\left[\frac{\left(\rho_{1}^{2}+\rho_{2}^{2}\right) \delta}{\delta-1}-1\right](1-k)^{2} \delta^{2}(T-t)^{3}+\int_{t}^{T}\left[a(\widehat{t})-\frac{\rho_{2} b \delta}{v(\delta-1)} u(\widehat{t})\right](1-k) \delta(T-\widehat{t}) \mathrm{d} \widehat{t}\right\}}}{\mathrm{e}^{\left\{\left(\delta k+\frac{\eta^{2} \lambda \mu^{2} \delta}{2 \sigma^{2}(\delta-1)}\right)(T-t)+\frac{\delta}{2(\delta-1) v^{2}} \int_{t}^{T} u(\widehat{t})^{2} \mathrm{~d} \hat{t}\right\}}} .
$$

In fact, if the deterministic function $a(t)=\widehat{a}>0$ and $u(t)=\widehat{u}>0$, where $\widehat{a}$ and $\widehat{u}$ are constants, $f(t)$ can be given in more explicit and simple form

$$
f(t)=\frac{\mathrm{e}^{\left\{\left[\frac{\eta \mu \delta^{2} \rho_{1} b \sqrt{\lambda}(1-k)}{2 \sigma(\delta-1)}+\left(\widehat{a}-\frac{\rho_{2} b \delta}{v(\delta-1)} \widehat{u}\right) \frac{(1-k) \delta}{2}\right](T-t)^{2}+\frac{b^{2}}{6}\left[\frac{\left(\rho_{1}^{2}+\rho_{2}^{2}\right) \delta}{\delta-1}-1\right](1-k)^{2} \delta^{2}(T-t)^{3}\right\}}}{\mathrm{e}^{\left\{\left(\delta k+\frac{\eta^{2} \lambda \mu^{2} \delta}{2 \sigma^{2}(\delta-1)}+\frac{\delta \hat{u}^{2}}{2(\delta-1) v^{2}}\right)(T-t)\right\}}} .
$$

Plugging (A10) into (A2) and (A3), respectively. The optimal strategy $\left(p^{*}, \pi^{*}\right)$

$$
\begin{aligned}
p^{*} & =-\frac{\eta \lambda \mu H_{x}+\rho_{1} b \sqrt{\lambda} \sigma H_{x r}}{\lambda \sigma^{2} H_{x x}} \\
& =-\frac{\eta \lambda \mu f(t) \mathrm{e}^{h(t) r} \delta x^{\delta-1}+\rho_{1} b \sqrt{\lambda} \sigma f(t) \mathrm{e}^{h(t) r} \delta x^{\delta-1} h(t)}{\lambda \sigma^{2} f(t) \mathrm{e}^{h(t) r} \delta(\delta-1) x^{\delta-2}} \\
& =-\frac{\eta \lambda \mu x+\rho_{1} b \sqrt{\lambda} \sigma x h(t)}{\lambda \sigma^{2}(\delta-1)} \\
& =-\frac{\eta \lambda \mu x+\rho_{1} b \sqrt{\lambda} \sigma x(1-k) \delta(T-t)}{\lambda \sigma^{2}(\delta-1)}, \\
\pi^{*} & =-\frac{u(t) H_{x}+\rho_{2} b v H_{x r}}{v^{2}(1-k) x H_{x x}} \\
& =-\frac{u(t) f(t) \mathrm{e}^{h(t) r} \delta x^{\delta-1}+\rho_{2} b v f(t) \mathrm{e}^{h(t) r} \delta x^{\delta-1} h(t)}{v^{2}(1-k) x f(t) \mathrm{e}^{h(t) r} \delta(\delta-1) x^{\delta-2}} \\
& =-\frac{u(t)+\rho_{2} b v h(t)}{v^{2}(1-k)(\delta-1)} \\
& =-\frac{u(t)+\rho_{2} b v(1-k) \delta(T-t)}{v^{2}(1-k)(\delta-1)} \\
& =-\frac{u(t)}{v^{2}(1-k)(\delta-1)}-\frac{\rho_{2} b \delta(T-t)}{v(\delta-1)} .
\end{aligned}
$$

The value function

$$
H(t, x, r)=\frac{\mathrm{e}^{\left\{\frac{\eta \mu \delta^{2} \rho_{1} b \sqrt{\lambda}(1-k)}{2 \sigma(\delta-1)}(T-t)^{2}+\frac{b^{2}}{6}\left[\frac{\left(\rho_{1}^{2}+\rho_{2}^{2}\right) \delta}{\delta-1}-1\right](1-k)^{2} \delta^{2}(T-t)^{3}\right\}} \cdot \mathrm{e}^{r(1-k) \delta(T-t)} x^{\delta}}{\mathrm{e}^{\left\{\left(\delta k+\frac{\eta^{2} \lambda \mu^{2} \delta}{2 \sigma^{2}(\delta-1)}\right)(T-t)+\frac{\delta}{2(\delta-1) v^{2}} \int_{t}^{T} u(\widehat{t})^{2} \mathrm{~d} \widehat{t}-\int_{t}^{T}\left[a(\widehat{t})-\frac{\rho_{2} b \delta}{v(\delta-1)} u(\widehat{t})\right](1-k) \delta(T-\widehat{t}) \mathrm{d} \widehat{t}\right\}}} .
$$

